\title{
Tabularia
}

TABULARIA Sources écrites des mondes normands médiévaux Les « Précieux Sangs » : reliques et dévotions | 2009

\section{Les romans du Graal et le culte du Précieux Sang}

Grail Literature and the Worship of the Precious Blood

\section{Edina Bozoky}

\section{OpenEdition \\ Journals}

Édition électronique

URL : http://journals.openedition.org/tabularia/1108

DOI : 10.4000/tabularia. 1108

ISSN : $1630-7364$

Éditeur :

CRAHAM - Centre Michel de Boüard, Presses universitaires de Caen

\section{Référence électronique}

Edina Bozoky, «Les romans du Graal et le culte du Précieux Sang », Tabularia [En ligne], Les « Précieux Sangs » : reliques et dévotions, mis en ligne le 08 juillet 2009, consulté le 01 mai 2019. URL : http:// journals.openedition.org/tabularia/1108; DOI : 10.4000/tabularia.1108 


\title{
Les romans du Graal et le culte du Précieux Sang Grail Literature and the Worship of the Precious Blood
}

\author{
Edina BоzокY \\ Université de Poitiers, CESCM \\ edina.bozoky@univ-poitiers.fr
}

Résumé:

Plusieurs thèmes de la littérature du Graal reflètent la dévotion à la Passion du Christ, et plus spécialement au Saint Sang. Après avoir esquissé l'histoire des romans français du Graal (fin $\mathrm{XII}^{\mathrm{e}}$-XIII ${ }^{\mathrm{e}}$ siècle), cette communication présente l'identification du Graal et de la lance-quisaigne avec des reliques de la Passion du Christ (Graal: plat de la Cène dans lequel Joseph d'Arimathie a recueilli le sang du Christ; la lance-qui-saigne: lance par laquelle Longin a transpercé le côté du Christ), puis les visions graaliennes qui mettent en scène le dogme de la transsubstantiation, étroitement lié au culte du sang du Christ.

Mots-clés: Romans du Graal, Saint Sang, Graal, lance-qui-saigne, Longin, Cène, Joseph d'Arimathie, Eucharistie, transsubstantiation, reliques de la Passion.

\begin{abstract}
:
Several themes in Grail literature reflect the devotion to the Passion of Christ, and especially to the Holy Blood. After outlining the development of French Grail romances (late $12^{\text {th }}$ to $13^{\text {th }}$ centuries) this paper will set out the ways in which the Grail and the Bleeding Lance came to be identified with relics from the Passion of Christ (the Grail as a dish from the Last Supper in which Joseph of Arimathea received Christ's blood; the Bleeding Lance as the lance with which Longinus pierced Christ's side). Finally, visions of the Grail will be presented as the enactment of the dogma of transubstantiation, which is closely linked to the religious significance of the Blood of Christ.
\end{abstract}

Keywords: Grail romances, Holy Blood, Grail, Bleeding Lance, Longinus, Last Supper, Joseph of Arimathea, Eucharist, transubstantiation, relics of the Passion.

La littérature du Graal est née à la fin du XII ${ }^{e}$ siècle et connut son plus grand essor durant la première moitié du XIII siècle, en particulier dans son premier tiers. Il s'agit d'une série de romans, d'abord en vers, puis en prose qui, en grande partie, s'organisent en cycle. Leur cadre est constitué par la cour du roi Arthur et les chevaliers de la Table Ronde, dont certains se lancent à la «quête» du Graal. Si les premières œuvres ont été rédigées en ancien français, et la production la plus riche reste en français, d'autres romans ont été composés en allemand, en anglais, en italien, en espagnol, en norois ${ }^{1}$.

1. Sur la littérature du Graal, la bibliographie est immense. Voir dernièrement: The Grail. A Casebook, éd. Dhira B. Mahoney, New York/Londres, Garland, 200o; Aurell, Martin, La légende $d u$ roi Arthur, Paris, Perrin, 2007, $692 \mathrm{p}$.

Tabularia «Études», n 9, 2009, p. 13-25, 8 juillet 2009 http://www.unicaen.fr/mrsh/craham/revue/tabularia/print.php?dossier=dossier9\&file=04bozoky.xml 
Les romans médiévaux du Graal ne peuvent pas être compris sans la connaissance du contexte de la religiosité médiévale des XII ${ }^{e}-\mathrm{XIII}^{e}$ siècles. En particulier, certains thèmes de cette littérature reflètent la dévotion à la Passion du Christ et plus spécialement le culte du Saint Sang.

\section{La littérature du Graal}

C'est dans le dernier roman de Chrétien de Troyes, intitulé Perceval ou le Conte $d u \mathrm{Graal}^{2}$, datable entre 1181 et 119o, que le thème du Graal apparaît. Le jeune héros du roman, qui ne «devine» son nom - Perceval - qu'après sa visite au château du Graal, rencontre un homme en train de pêcher dans une barque sur une rivière. Celui-ci lui offre l'hospitalité pour la nuit et lui indique le chemin qui mène vers sa «maison ». Perceval n'aperçoit pas immédiatement le château blotti au fond d'une vallée. Accueilli par son hôte, infirme des jambes, le héros assiste, durant le repas, à un cortège étrange: un jeune homme sort d'une chambre, portant une lance d'une blancheur éclatante; une goutte de sang perle à la pointe de la lance et coule jusqu'à la main du porteur. Puis viennent deux autres jeunes hommes, tenant des chandeliers d'or; ensuite apparaît une demoiselle, tenant un graal entre ses deux mains, un plat large et peu creux, tandis qu'une clarté extraordinaire se répand dans la salle; et enfin, une autre demoiselle arrive avec un tailloir (plat à découper) en argent. Bien qu'intrigué par cette scène, le jeune héros garde le silence, se souvenant des conseils d'un vieux chevalier qui lui avait enseigné la discrétion. Le lendemain, il serait prêt à poser les questions : à qui serton le Graal? et pourquoi la lance saigne-t-elle? - mais il trouve le château complètement désert, et lorsqu'il part, le pont-levis se lève brutalement derrière lui ${ }^{3}$. Plus tard, il apprendra des bribes d'informations sur cette aventure. D'abord, une demoiselle lui fait des reproches véhéments: s'il avait posé des questions, il aurait guéri son hôte, le Roi-Pêcheur, blessé entre les jambes, et aurait empêché les malheurs qui frapperont sa terre ${ }^{4}$. Puis, au bout de cinq années d'errance, un vendredi saint, Perceval arrive chez un ermite qui se révèle être son oncle ainsi que celui du Roi-Pêcheur. Le saint homme lui donne un seul renseignement, quoique énigmatique, sur le Graal: c'est une «sainte chose» qui ne contient qu'une seule hostie et qui maintient en vie le vieux roi, père du Roi-Pêcheur ${ }^{5}$. Il ne dit rien sur la lance ${ }^{6}$. Mais dans un autre passage du roman, il est question d'une

2. Chrétien de Troyes, Perceval ou Le Conte du Graal, éd. et trad. Daniel Poirion, in Chrétien DE Troyes, Euvres complètes, Paris, Gallimard (coll. La Pléiade), 1994, p. 683-911; voir aussi la trad. de Lucien Foulet, in La légende arthurienne, Danielle RÉGNIER-Bohler (dir.), Paris, Laffont, 1989, p. 7-115; trad. de Charles MéLA in La légende du Graal dans les littératures européennes. Anthologie commentée, Michel Stanesco (dir.), Paris, Librairie générale française (coll. La Pochothèque), 2006, p. 125-284.

3. Chrétien de Troyes, Perceval..., v. 2998-3419, p. 759-770.

4. Ibid., v. 3494-3611, p. 772-774.

5. Ibid., v. 6337-6431, p. 841-843.

6. Sur la lance-qui-saigne, voir Brown, Arthur C. L., "The Bleeding Lance», Publications of the Modern Language Association of America, 25, 1920, p. 1-59. 
lance-qui-saigne qui, selon une prophétie, pourrait détruire le royaume de Logres (nom du royaume d'Arthur). C'est l'autre protagoniste du roman, Gauvain, qui est envoyé à sa quête, et ne fait pas grand-chose pour la trouver...

Le roman de Chrétien restant inachevé, le «secret » du Graal et de la lance ne pouvait pas être connu. C'est alors que plusieurs auteurs inventent soit une continuation, soit une "préhistoire» au roman de Perceval. Tout d'abord, à la fin du $\mathrm{XII}^{\mathrm{e}}$ siècle (ou début du XIII ${ }^{\mathrm{e}}$ ), un certain Robert de Boron, dans son roman en vers Joseph d'Arimathie ou le Roman de l'Estoire du Graal $^{7}$ établit un lien entre l'histoire apocryphe de la Passion du Christ et le Graal (sans s'intéresser au thème de la lance). Robert a écrit également un Merlin en vers, conservé seulement en fragments, et peut-être un Perceval dont il ne reste rien. La mise en prose ${ }^{8}$ de cette hypothétique dernière partie constitue une histoire du Graal christianisé qui en situe les épisodes en Bretagne, à l'époque de la chevalerie arthurienne. La trilogie Joseph-Merlin-Perceval relie, pour la première fois, la Terre Sainte au pays des Bretons insulaires, et combine les légendes apocryphes sur la Passion du Christ avec les traditions pseudo-historiques sur Arthur et avec la matière des contes merveilleux celtiques.

Dans le même temps, également à la fin du XII ${ }^{\mathrm{e}}$ siècle, sont composées les deux premières Continuations ${ }^{9}$ du roman de Chrétien, en reprenant le fil des aventures respectivement de Gauvain et de Perceval. Vers la fin du premier tiers $\mathrm{du}$ XIII ${ }^{\mathrm{e}}$ siècle, deux autres Continuations naissent (celles de Manessier ${ }^{10}$ et de Gerbert de Montreuil ${ }^{11}$ ).

C'est de cette époque que date aussi l'imposant cycle du Graal en prose, appelé cycle de la Vulgate ou Lancelot-Graal. Sa partie la plus célèbre est la Quête du saint Graal (1225-1230) ${ }^{12}$ dont le protagoniste, Galaad, est un chevalier pur et chaste, qualifié de celestiel, seul digne d'achever l'aventure du Graal.

7. Robert de Boron, Le Roman de l'Estoire dou Graal, éd. William A. Nitze, Paris, Champion (Les Classiques français du Moyen Âge), 1927, 136 p.; voir aussi Joseph d'Arimathie. A Critical Edition of the Verse and Prose Versions, éd. Richard O'Gorman, Toronto, Pontifical Institute of Medieval Studies, 1995, 575 p.; trad. d'Alexandre MichA, in La légende du Graal..., p. 303-357.

8. Didot-Perceval [selon le nom de l'ancien propriétaire du manuscrit de la BnF, fr. n.a. 4166], éd. William RoAch, Philadelphie, University of Pensylvania Press, 1941 et Genève, Slatkine Reprints, 1977,348 p.

9. Première Continuation ou Continuation-Gauvain: The Continuations of the Old French Perceval of Chrétien de Troyes, t. I-III: The First Continuation, éd. William Roach, Philadelphie, 1949-1952; trad. (de la rédaction courte) Colette-Anne VAN Coolput-STorms, Paris, Librairie générale française (Lettres gothiques), 1993. Deuxième Continuation ou Continuation-Perceval: The Continuations of the Old French Perceval of Chrétien de Troyes, t. IV : The Second Continuation, éd. William Roach, Philadelphie, The American Philosophical Society, 1971; trad. Simone Hannedouche, Perceval et le Graal. Les Continuations, Paris, Triades, 1968.

10. Manessier, La Troisième Continuation du Conte du Graal, éd. bilingue, publication, traduction, présentation et notes par Marie-Noëlle Toury, avec le texte édité par William RoAcH, Paris, Champion (Champion Classiques, Moyen Âge), 2004.

11. Gerbert de Montreuil, La Continuation de Perceval [Quatrième Continuation], éd. Mary Williams - Marguerite Oswald, Paris, Champion (Les Classiques français du Moyen Âge), $1922,1925,1975$.

12. La Quête du Saint Graal, roman en prose du XIII siècle, éd. Fanni BogDanow, trad. Anne BERRIE, Paris, Librairie générale française (Lettres gothiques), 2006, $831 \mathrm{p}$. 
Pour faire le lien avec les temps christiques mais aussi avec le monde arthurien, deux autres romans du cycle sont importants: Estoire del Saint Graal (vers 1230$1235)^{13}$ et le Lancelot "propre» (vers 1220-1225) ${ }^{14}$. Ce cycle sera en partie refondu et incorporé dans le Tristan en prose ${ }^{15}$, immense roman-fleuve très populaire à la fin du Moyen Âge.

Il faut également mentionner un roman en prose à part, le Perlesvaus ou le Haut livre du Graal (datation discutée: début du XIII ${ }^{\mathrm{e}}$ siècle, ou après 1230) ${ }^{16}$, caractérisé par une atmosphère assez curieuse, et dont le thème principal est la lutte de la Vieille Loi (le paganisme) et de la Nouvelle (le christianisme). Perceval et ses compagnons y mènent une véritable croisade pour imposer le christianisme.

Plusieurs aspects du thème du Graal ont un lien étroit avec le culte du Saint Sang.

\section{Le Graal et la lance-qui-saigne, reliques de la Passion}

Premièrement, les deux objets énigmatiques qui apparaissent dans le Perceval de Chrétien de Troyes deviennent des reliques contenant ou conservant le Saint Sang du Christ.

Robert de Boron ne mentionne que le Graal dans son roman inspiré des apocryphes de la Passion (l'Évangile de Nicodème ${ }^{17}$, connu aussi sous le nom de Gesta Pilati; Vindicta Salvatoris ${ }^{18}$ ). Il identifie le Graal avec un plat (veissel) utilisé lors de la Cène. Lors de l'arrestation du Christ, un Juif l'emporte et l'offre à Pilate, qui le donne à Joseph d'Arimathie par amitié. Lorsque Joseph et Nicodème descendent de la croix le corps du Christ, Joseph le lave, et il voit le sang s'écoulant de ses plaies. Il court alors prendre le veissel pour y recueillir le sang. Ensuite Joseph enveloppe le corps du Christ dans une étoffe et le dépose dans un sépulcre de pierre. Plus tard, Joseph d'Arimathie est emprisonné mais, miraculeusement, Dieu lui apporte à la prison le veissel que Joseph avait caché dans sa maison. À sa vue, Joseph est rempli de la grâce du Saint-Esprit. Dieu (en fait,

13. L'Estoire del Saint Graal, éd. Jean-Paul Ponceau, Paris, Champion (Classiques français du Moyen Âge), 1997, 2 vol. p. 120-121.

14. Lancelot, éd. Alexandre Micha, 1978-1983, 9 vol. (Textes littéraires français); trad. partielle par Alexandre Micha, Paris, 10/18, 1984, 2 vol

15. Le Roman de Tristan en prose, t. I-IX, Genève, Droz (Textes littéraires français), 1987-1997.

16. Perlesvaus ou le Haut Livre du Graal, éd. William A. Nitze - T. Atkinson Jenkins, Chicago, 1932-1937; trad. fr. par Anne Berthelot, Greifswald, Reineke-Verlag, 1997.

17. Evangelia apocrypha, éd. Constantin Tischendorf, Leipzig, Avenarius \& Mendelssohn, 1853; $1876^{2}$, p. LIV-LXXVII et 210-434; KIm, H. C., The Gospel of Nicodemus, Gesta Salvatoris, Toronto, 1973; trad. fr. Rémi GounelLe, Zbigniew IzyDorczyк, L’Évangile de Nicodème ou les Actes faits sous Ponce Pilate (recension latine A), suivi de La lettre de Pilate à l'empereur Claude, Turnhout, Brepols, 1997; trad. Ch. Fourrer, présentation par Rémi GounelLe, in Écrits apocryphes chrétiens, II, éd. Pierre Geoltrain et Jean-Daniel Kaestui, Paris, Gallimard (Coll. la Pléiade), 2005, p. 249-297.

18. Evangelia apocrypha..., p. LXXXII-LXXXIV et 471-486; trad. fr. G. Besson, Michèle BrossARDDANDRÉ et Zbigniew IzYDORCZYK, in Écrits apocryphes chrétiens..., p. 369-398. 
Jésus-Christ) confie la garde du récipient à Joseph et lui explique comment célébrer une cérémonie eucharistique que Joseph devra accomplir avec le plat ${ }^{19}$.

L'identification de la lance-qui-saigne a lieu dans les Continuations du roman de Chrétien: dans la Continuation-Perceval, Gauvain apprend par le RoiPêcheur que c'est la lance dont le Christ avait été transpercé sur la croix (ms E, v. 31188 sq.). Dans la Continuation-Gauvain, version longue, c'est aussi Gauvain qui obtient l'information. Mais ici, lors de la seconde visite de Gauvain au château du Graal, la lance n'est pas portée en cortège; elle est fichée dans un vase (orcel) d'argent, saignant à foison; les éclaboussures des gouttes de sang sont visibles tout autour du récipient. Le sang vermeil est conduit par un tuyau (tuël) d'or dans un conduit d'émeraude qui sort de la salle. Le roi raconte à Gauvain que c'est la lance dont le Christ avait été transpercé à la croix; depuis, elle se trouve toujours au château du roi; elle saigne continûment et saignera jusqu'au jour du Jugement dernier (v. 17396 sq.). Ce jour-là, tout le monde verra le Créateur verser du sang aussi frais que le fait actuellement la lance. Selon cette Continuation, Joseph d'Arimathie a fait fabriquer le Graal en or pur, et le jour de la Crucifixion, il y a recueilli le sang du Christ qui coulait de ses blessures. Il plaça ensuite le Graal dans une armoire précieuse et il y priait chaque jour pour vénérer le Saint Sang.

L'essentiel de la christianisation du Graal et de la lance se fait donc peu après la mort de Chrétien de Troyes. Tout en gardant des traits qui rappellent les objets merveilleux du conte populaire, ils sont désormais des reliques contenant ou conservant le Saint Sang. Ce processus d'identification reflète l'essor du culte des «instruments» de la Passion du Christ. Sur le modèle des récits de translations de reliques, les romans du Graal racontent aussi les itinéraires aventureux du Graal et de la lance, avec plus de détails dans le cas du Graal que dans celui de la lance. De même, à la manière des reliques, le Graal et la lance accomplissent des miracles, en particulier des miracles de guérison. Toutefois nous devons souligner que, même dans les romans les plus «christianisés», le Graal présente des aspects magiques. C'est ainsi que, dans la Première Continuation, le Graal est dispensateur de nourritures et de boissons terrestres: en cas de besoin, Joseph prie Dieu et le Graal arrive et dépose partout du vin et des mets en abondance au gré de chacun! Il en est ainsi lors de la visite de Gauvain au château mystérieux d'un roi: le Graal sert le pain et le vin et tous les mets (p. 295). De même, dans la Continuation de Gerbert de Montreuil, Joseph d'Arimathie et ses compagnons, jetés en prison, sont nourris par le Graal (v. 44678 sq.). Dans d'autres romans, le motif du vase d'abondance est influencé par le miracle évangélique de la multiplication des pains et des poissons. Dans un épisode de L'Estoire, Josephé, fils de Joseph d'Arimathie, et ses compagnons - plus de cinq cents personnes - reçoivent douze pains qui ne suffisent pas à tout le monde. Josephé fait alors partager les pains en trois et fait apporter le Graal: après quoi, les douze pains non seulement rassasieront tout le monde, mais il en restera même ${ }^{20}$. Dans la Queste, le Graal apparaît le jour de la Pente-

19. Robert De Boron, Le Roman..., v. 395-398; 433-435; 507-509; 555-574; 713-734; 851-870.

20. L'Estoire del Saint Graal..., t. II, \$672-673, p. 426-427. 
côte à la cour d'Arthur et, passant devant les tables, dépose devant chaque chevalier des mets que celui-ci désire ${ }^{21}$.

Cependant c'est l'aspect relique qui domine dans ces romans. Soulignons qu'à ces reliques de la fiction correspondent des objets réels, dont certains sont attestés dès le haut Moyen Âge. La légende de Longin et de sa lance a fortement influencé le thème de la lance-qui-saigne dans la littérature du Graal ${ }^{22}$. L'arme par laquelle Longin avait transpercé le côté du Christ, était une relique précieuse, considérée comme l'un des instruments du salut. Selon une source byzantine, la Chronique pascale, sa pointe est arrivée de Jérusalem à Constantinople au début du VII ${ }^{e}$ siècle ${ }^{23}$. Mais vers 670, selon Arculfe, rapporté par Adamnan, cette lance est toujours conservée à Jérusalem, dans la basilique constantinienne du Martyrium, élevée sur l'emplacement de l'invention de la Croix ${ }^{24}$. Au $\mathrm{X}^{\mathrm{e}}$ siècle, Constantin Porphyrogénète parle de la cérémonie de vénération de la lance le Vendredi saint. Elle se trouve alors à la chapelle de la Vierge du Phare du Palais ${ }^{25}$. C'est de cette même relique que saint Louis obtient un morceau en $1242^{26}$. Mais une autre a été aussi découverte en 1098 à Antioche, et a joué le rôle d'excitatorium dans l'élan des croisés à la poursuite de leur chemin jusqu'à Jérusalem ${ }^{27}$. La lance disparaît de la documentation dès le début du XII ${ }^{e}$ siècle.

Il est important de noter que, dès le XII ${ }^{e}$ siècle au moins, apparaissent des plats que l'on dit avoir servi à la Cène au repas du Seigneur, bien qu'il ne semble

21. La Quête du Saint Graal..., \$19, p. 110-113. Voir sur ce sujet: BAUdRY, Robert, "La vertu nourricière du Graal», in Banquets et manières de table au Moyen Âge, Senefiance 38, Aix-en-Provence, Publications de l'Université de Provence, Cuerma, 1995, p. 433-450 et Berthelot, Anne, «Le Graal nourricier», ibid., p. 451-466.

22. Voir sur ce sujet: Peebles, Rose Jeffries, The Legend of Longinus in Ecclesiastical Tradition and English Literature, and its Connection with the Grail, Baltimore, J. H. Furst, 1911; BurdACH, Konrad, Der Gral. Forschungen über seinen Ursprung und seinen Zusammenhang mit der Longinuslegende, Stuttgart, Kohlhammer, 1938.

23. Voir sur la date de son arrivée d'après le Chronicon Paschale, KLEIN, Holger A., "Constantin, Helena, and the Cult of the True Cross in Constantinople», in Byzance et les reliques du Christ, Jannic Durand et Bernard Fuusin (éd.), Paris, Association des Amis du Centre d'Histoire et Civilisation de Byzance, 2004, p. 31-59, ici p. 42 et 45.

24. Adamnan, De locis sanctis, I, VIII, éd. Ludwig BIELER, Itineraria et alia geographica, Turnhout, Brepols (Corpus Christianorum, Series Latina CLXXV), 1965, p. 191-192; trad. Pierre Maraval, Récits des premiers pèlerins chrétiens au Proche-Orient (IV $V^{e} V I I^{e}$ siècle), Paris, Cerf, 1996, p. 247 : "Arculfe a vu également la lance du soldat avec laquelle celui-ci avait frappé le côté du Seigneur suspendu sur la croix. Cette lance se trouve dans le portique de la basilique de Constantin, sertie dans une croix de bois. Sa hampe est divisée en deux parties.»

25. Constantin Porphyrogenete, Liber de ceremoniis, I, 24, Patrologia Graeca, éd. Jacques-Paul Migne, 112, col. 421-422.

26. Voir le catalogue Le trésor de la Sainte-Chapelle, Jannic Durand et Marie-Pierre Laffitte (dir.), Paris, Réunion des Musées Nationaux, 2001, p. 82-84 et passim.

27. Voir Runciman, Steven, "The Holy Lance found at Antioch», Analecta Bollandiana, 68, 1950, p. 197-209. Sur la Lance de Constantinople et d'Antioche, voir aussi De Mely, Fernand, «Reliques de Constantinople. La Sainte Lance», Revue de l'Art chrétien, $40^{\mathrm{e}}$ année, $4^{\mathrm{e}}$ série, t. VIII, 1897, p. 1-11 et 120-127. 
pas possible d'établir un lien direct entre ces «reliques» et la genèse du thème du Graal. Le Sacro Catino de Gênes (conservé dans le trésor de la cathédrale), a été rapporté par Guglielmo Embriaco («Tête de Maille») de la Terre sainte, soit en 1101 de Césarée de Palestine, soit en 1099 d'un temple construit par Hérode le Grand à Jérusalem. En réalité, c'est une coupe en émeraude, une œuvre islamique du IX ${ }^{e}$ siècle (?) ${ }^{28}$. C'est seulement Jacques de Voragine, archevêque de Gênes (1230-1298) qui l'identifie au saint Graal «selon quelques livres anglais ${ }^{29}$ ".

Le Saint Calice de Valence fut aussi considéré comme le plat de la Cène. Selon la légende, il aurait été apporté de Jérusalem à Rome par saint Pierre. Sous la persécution de l'empereur Valérien, il aurait été confié au diacre saint Laurent, qui l'a envoyé en Espagne, dans l'église de Huesca. À cause des invasions arabes, il a été transféré à San Juan de la Peña où il est resté jusqu'en 1399, puis est passé à la chapelle royale de Saragosse. Il est conservé depuis 1439 à la cathédrale de Valence ${ }^{30}$.

Dans le même temps, bien qu'un assez grand nombre de reliques du Saint Sang soient attestées en Orient et en Occident, leurs récipients mêmes ne furent pas particulièrement vénérés.

Si l'on se tourne maintenant vers les "cérémonies " graaliennes, on constate que la symbolique du Graal et en partie celle de la lance se réfèrent davantage au culte eucharistique qu’à la vénération des reliques du Saint Sang.

\section{La transsubstantiation et les visions graaliennes}

Le Graal devient également symbole du sacrement de l'Eucharistie et de la grâce du Saint-Esprit, et les liturgies graaliennes mettent en scène des visions frappantes de la présence réelle et de la transsubstantiation, dans lesquelles le motif du sang du Christ occupe une place importante.

Déjà dans le roman de Robert de Boron, Dieu enseigne à Joseph comment faire le «service du Graal» - c'est-à-dire la célébration du rite eucharistique: «Tu m'as enlevé de la croix et tu m'as couché dans le sépulcre: ces faits sont symbolisés par l'autel sur lequel me mettront ceux qui me sacrifieront. Le linge où je fus enveloppé sera appelé corporal. Le vase dans lequel tu as mis mon sang, quand tu l'as recueilli de mon corps, sera appelé calice. La patène qui sera placée dessus représentera la pierre scellée sur moi, quand je fus mis au tombeau... ${ }^{31}$ ».

28. Voir dernièrement Müller, R., «Il Sacro Catino», in Intorno al Sacro Volto, da Bisanzio a Genova, Anna Rosa Calderoni Masetti, Colette Dufour Bozzo et Gerhard Wolf (dir.), Venise, Marsilio, 2007, 250 p.

29. Voir Jacopo di Voragine e la sua Cronaca di Genova, éd. Giovanni Monleone, Rome, Istituto Storico Italiano, 1941, t. I, p. 307-314.

30. Voir Onate Ojeda Juan Angel, El Santo Grial. El Santo Caliz de la Cena, venerado en la Sta. Iglesia Catedral Basilica Metropolitana de Valencia, Valencia, Nàcher, 1990; SAnCHez Navarrete, Manuel, El Santo Caliz de la Cena, (Santo Grial) venerado en la Catedral de Valencia, Valencia, Cofradria del Santo Càliz, 1994; Sancho Andreu, Jaime, éd., El Santo Caliz. Historia, leyenda y culto, Valencia, Generalitat Valenciana, 2006.

31. Robert de Boron, Le Roman..., v. 893-916. 
Mais c'est surtout dans L'Estoire et la Queste que le lien entre le sacrifice du Sauveur, l'Eucharistie et le Graal est établi d'une façon visionnaire. Dans L'Estoire, qui constitue la préhistoire de l'aventure du Graal, Joseph d'Arimathie emporte le Graal (appelé ici escuelle) dans une arche (coffre) dans le royaume de Sarras, en Orient. C'est ici qu'ont lieu l'ordination et le sacre de Josephé par Jésus-Christ, au milieu d'une série de visions miraculeuses. Lorsque Josephé ouvre l'arche - qui tout à coup s'agrandit alors quatre fois de sa taille -, il voit d'abord une présentation animée des instruments (ou les "armes du Christ»: Arma Christi) de la Passion, tenus par des anges: la croix, trois clous (de la crucifixion), la lance, l'éponge et le fouet. En deuxième «tableau », il est témoin du percement par la lance du côté de l'homme crucifié; sortant de la plaie, le sang et l'eau coulent dans l'écuelle (le Graal) que son père avait déposée dans l'arche. Plus tard, Josephé voit, posés sur un autel, les trois clous et le fer de la lance, tout sanglants, ainsi que l'écuelle et, au milieu de l'autel, un riche vaissiel d'or recouvert d'un couvercle d'or et d'un tissu blanc. Ensuite, les anges apportent un récipient plein d'eau, un jetoir (goupil), des toiles blanches, des encensoirs. Apparaît Jésus-Christ en personne, et un ange asperge d'eau tous les assistants. Jésus-Christ explique à Josephé que c'est l'eau de la purification, pour nettoyer le lieu de tout mauvais esprit. Dans cette série de scènes, les instruments de la Passion sont animés - avec une véritable reconstitution d'un moment de la Passion -, et sont mis en relation directe, sur l'autel, avec le sacrement de l'Eucharistie ${ }^{32}$. Il s'agit, entre autres, d'une évocation précoce de la «messe de saint Grégoire»: en effet, la représentation de la vision des instruments de la Passion devant un célébrant de la messe - en l'occurrence, saint Grégoire - est un thème iconographique de la fin du Moyen Âge ${ }^{33}$.

Dans la Queste, le Graal apparaît à plusieurs reprises, soit en rêve, soit en vision. C'est la scène finale du roman qui contient la vision la plus extraordinaire ${ }^{34}$. Galaad, le chevalier élu de l'aventure et les chevaliers dignes assistent au château de Corbenic à une liturgie à la fois céleste et réelle. La messe est célébrée par l'évêque Josephé: quatre anges l'assistent, dont deux portent des cierges, le troisième, un linge de samit (soie) vermeil, et le quatrième tient la lance dont le sang coule dans une boîte qu'il porte dans son autre main; ensuite, il fait couler le sang dans le Graal. Josephé lève la lance et couvre le Graal de la touaille. Il prend une hostie en semblance de pain dans le Graal. Quand il le lève, un enfant au visage de feu descend du ciel. Le miracle de la transsubstantiation s'accomplit alors: l'enfant entre dans le pain et tous ceux qui sont dans la salle voient très distinctement que le pain prend la forme d'un homme charnel. Après l'avoir tenu un long moment, Josephé le repose dans le Graal. Ensuite, Josephé annonce aux chevaliers qu'ils seront nourris par le Sauveur en personne de la «nourriture la plus sainte et la plus douce». Il disparait, et sort alors du saint vaissel un

32. L'Estoire del Saint Graal..., t. I, $\$ 111-120$, p. 72-78.

33. Voir Vloberg, Maurice, L'Eucharistie dans l'art, Grenoble/Paris, Arthaud, 1946, t. II, p. 199-208.

34. La Quête du Saint Graal..., \$320-323, p. 632-639. 
homme tout nu, dont les mains, les pieds et le corps sont tout sanglants - le Christ. C'est lui qui donne la communion aux chevaliers, puis il explique à Galaad que le Graal est l'écuelle dans laquelle Jésus-Christ avait mangé l'agneau le jour de Pâques. Ensuite, il ordonne à Galaad de l'emporter à la cité de Sarras, au Palais spirituel. Mais avant de partir, il doit guérir le Roi Méhaigné («blessé»), en oignant ses jambes et ses cuisses avec le sang de la lance.

À la fin du roman, au Palais Spirituel, une nouvelle célébration mi-céleste mi-terrestre a lieu autour du Graal que Galaad dépose sur une table d'argent et recouvre d'une arche d'or et de pierres précieuses. Un homme vêtu en évêque, entouré d'anges, célèbre la messe; lorsqu'il ôte la patène couvrant le Graal, il invite Galaad à regarder à l'intérieur du vase. Il devient alors témoin des mystères célestes. Il meurt peu après, et une main qui descend du ciel emporte alors le Graal et la lance ${ }^{35}$.

Je n'ai évoqué ici que les passages les plus significatifs de la «liturgie»du Graal. Ils ont été écrits à l'époque où la transsubstantiation est devenue dogme officiel (1215, $\mathrm{IV}^{\mathrm{e}}$ concile œcuménique de Latran). Dans la littérature édifiante, un grand nombre de miracles racontent dès le XII ${ }^{e}$ siècle la révélation de la vraie nature de l'Eucharistie, sous la forme de l'enfant Jésus, le Christ souffrant, etc. (écrits de Pierre le Vénérable et d'Herbert de Clairvaux; Conrad d'Eberbach, Exordium magnum ordinis cisterciensis, 1193-1221; Césaire de Heisterbach, Dialogus miraculorum, 1223-24, etc. ${ }^{36}$. Mais avant même que le culte eucharistique atteigne son apogée ${ }^{37}$, il est remarquable que, dans les romans du cycle de la Vulgate, nous trouvons toute une panoplie d'images particulièrement évocatrices se rapportant à la présence réelle: l'apparition de l'enfant, du Christ de pitié, mais aussi celle des instruments de la Passion, avec la vision du sang sacrificiel du Christ crucifié. On doit souligner que, dans la Queste, l'accent est mis sur le caractère proprement eucharistique du Graal, et le culte du Précieux Sang est ramené à la lance. Cependant, le Graal de la Queste, apparaissant un certain nombre de fois et faisant divers miracles, ne peut pas être réduit à une manifestation du sacrement eucharistique : avec Albert Pauphilet, on peut dire que «tous les attributs du Graal sont ceux mêmes de Dieu. [...] il a la toute-puissance et la grâce miraculeuse »; "Li sains Graaus, ce est la grace del Saint Esperit,» dit un ermite. «Plus exactement, le Graal, c'est la manifestation romanesque de Dieu ${ }^{38} »$. De cette façon, «la quête du Saint Graal, les aventures du Saint Graal, c'est l'histoire des âmes à la recherche de Dieu... ${ }^{39}$ ".

D'autres épisodes des romans du Graal sont également associés à la symbolique eucharistique et au Saint Sang. Évoquons notamment l'aventure de

35. Ibid., $\$ 330-332$, p. $650-653$.

36. Voir la synthèse de Rubin, Miri, Corpus Christi. The Eucharist in Late Medieval Culture, Cambridge, Cambridge University Press, 1991, XV-432 p.

37. La fête du Corpus Domini devient officielle en 1264 .

38. Pauphilet, Albert, Études sur la Queste del Saint Graal, Paris, Champion, 1921, réimp. 1980, p. 24-25.

39. Ibid., p. 26. 
la Bête glatissant ${ }^{40}$. Dans le Perlesvaus, le héros arrive un jour dans une belle lande, au milieu de la Forêt soutaine («reculée»). Il y aperçoit une croix vermeille, et un beau chevalier, vêtu de blanc et tenant un vessel d'or ainsi qu'une très belle demoiselle, également vêtue de blanc, et tenant aussi un vessel d'or. Survient alors la Bête glatissant, une bête hybride, toute blanche, très belle, plus petite qu'un renard. On entend le glapissement de douze chiots dans son ventre... Affolée, la bête court vers la croix et met bas ses chiots, qui la tuent et la dépècent, mais ne la mangent pas. Alors le chevalier et la demoiselle prennent les morceaux de sa chair et les mettent dans leurs récipients, adorent la Croix et s'en vont. Une odeur suave se répand à cet endroit. Il semble évident que cet épisode est une transposition assez curieuse du sacrifice du Christ et du thème de l'Eucharistie.

\section{Blessures et guérisons}

Un troisième thème, celui de la blessure du Roi-Pêcheur et de ses doublets, a un rapport plus ou moins étroit avec le sang du Christ, par le biais du Graal ou de la lance. Depuis Chrétien de Troyes, ce thème est fondamentalement associé à l'aventure du Graal. Selon le Perceval de Chrétien, si Perceval avait posé les questions sur le Graal et la lance, le Roi-Pêcheur, infirme à la suite d'une blessure entre les hanches, aurait guéri; sans cela, il reste méhaigné, et son pays, dévasté. Dans quelques autres romans du Graal, la blessure du roi, liée parfois à la fertilité de son pays, conserve un caractère archaïque, non chrétien. Mais dans les romans du cycle de la Vulgate, les blessures des rois, parfois gardiens du Graal, constituent le contrepoint de la blessure du Christ. Dans L'Estoire et la Queste, la guérison de la blessure, produite par le sang de la lance-qui-saigne, se réfère au salut de l'humanité par le Christ. Dans un épisode de L'Estoire, un ange guérit si bien la blessure de Josephé avec le sang qui a coulé de la lance qu'aucune trace n'en reste ${ }^{41}$. Nous avons évoqué comment Galaad guérit le roi en l'oignant avec le sang de la lance dans la Queste ${ }^{42}$. Il est curieux que, sur un dessin d'un manuscrit de la Queste, Galaad touche la jambe du roi avec le Graal, en forme d'un calice, comme si l'instrument de la guérison était le récipient même (ms BnF fr. 343 , f. 103) ... ${ }^{43}$.

La littérature graalienne témoigne que les préoccupations majeures de la dévotion, de la piété, voire de la théologie peuvent imprégner même des œuvres de fiction, dont le principal public appartenait à la chevalerie, à la société de la

40. Voir sur ce thème Bozoky, Edina, «La Bête glatissant et le Graal», Revue d'histoire des religions, 188, 1974, p. 127-148; Roussel, Claude, «Le jeu des formes et des couleurs: observations sur la 'beste glatissant'», Romania, 104, 1983, p. 49-82.

41. L'Estoire del Saint Graal..., t. I, $\$ 268$, p. 166.

42. La Quête du Saint-Graal..., \$323, p. 638-639.

43. Voir Stones, Alison, "Seeing the Grail. Prolegomena to a Study of Grail Imagery in Arthurian Manuscripts », in The Grail. A Casebook..., p. 301-366, ici p. 333. 
cour. Mais toutes ces transpositions littéraires de thèmes religieux n'ont pas réussi pour autant à transformer les romans du Graal en écrits didactiques: l'Église se méfiait de cette légende qu'elle n'a jamais admise comme authentique. Toujours est-il que la variété des aventures, la diversité des personnages, la richesse des motifs merveilleux en font des œuvres fascinantes dont la popularité perdure jusqu'à nos jours.

\section{Conclusions de la Table ronde}

Les communicants à ce colloque ont brossé les divers aspects du thème du Précieux Sang, de ses aspects généraux à l'analyse des manifestations spécifiques de son culte et de ses reliques. De même, nous avons pu parcourir, dans la durée, jusqu'à notre époque où le culte des reliques garde son actualité comme Cyril Brun l'a souligné, tout en s'interrogeant sur le sens de ces dévotions aujourd'hui.

André Vauchez a mis en relief l'intérêt actuel pour l'histoire des reliques: depuis une petite trentaine d'années, les chercheurs revoient l'importance des reliques dans l'histoire, leur fonction même dans l'exercice du pouvoir, au-delà de leur rôle religieux.

Jacques Le Maho a permis de «revisiter» l'histoire des textes sur Fécamp en ouvrant la voie à de nouvelles recherches, en exprimant quelque doute sur l'existence d'un pèlerinage avant la fin du XII ${ }^{\mathrm{e}}$ siècle. Il a montré le lien entre reconstruction et réinvention d'une relique, un dossier dont l'exploration devrait être continuée.

Nicholas Vincent s'est attaché au rapport quelque peu incertain entre les reliques de Fécamp et de Norwich dans une passionnante communication. Lucile Tran-Duc a mis en contexte le dossier de Fécamp, en le comparant avec les autres reliques célèbres qui apparaissent avant 1204, et en soulignant leur lien privilégié avec les grands laïcs.

Puis nous avons fait le tour des interprétations symboliques et spirituelles du sang du Christ par le biais d'un thème iconographique - des anges autour de la Croix - avec la communication de Christine Pellistrandi.

L'évocation de la littérature du Graal, étroitement liée aux objets-reliques du sang du Christ, a permis de mesurer l'impact des préoccupations religieuses de la fin XII ${ }^{\mathrm{e}}$-début XIII ${ }^{\mathrm{e}}$ siècle sur la fiction des romans arthuriens.

Enfin, nous avons abordé la relation du Saint Sang avec la théologie de l'Eucharistie (Pascal Pradié, "Controverses eucharistiques»; Marc Vénard, "Sang eucharistique - sang relique»). Avec la communication de Nicolas Leroux, qui a évoqué la pratique du pèlerinage au $\mathrm{XIX}^{\mathrm{e}}$ siècle, nous sommes presque arrivés au seuil de notre époque...

Pour terminer mes rapides conclusions, je voudrais faire part de quelques réflexions autour du thème du Saint Sang.

Depuis les plus anciennes civilisations, le sang représente l'essence même de la vie, il véhicule la vie. Au Moyen-Orient, le sang était considéré comme 
un archétype de l'âme humaine; dans la Bible, Iahvé dit à Moïse que l'âme de la chair est dans le sang. De même, dans la médecine antique, le sang est associé au souffle vital.

Dans le christianisme, curieusement, le Saint Sang, celui du Christ, n'est pas l'objet des plus importantes vénérations, et son culte se développe plutôt tardivement. Pourtant, le sang des martyrs figure parmi les principaux attributs de la sainteté dès le $I^{\mathrm{e}}$ siècle. Toute une série de références pourraient être évoquées, indiquant que la fraîcheur du sang des martyrs, ou le sang qui se remet à couler de leur blessure bien après leur mort, attestent qu'ils sont toujours vivants dans leurs restes corporels. Ces phénomènes miraculeux sont la manifestation du pouvoir divin, de la virtus. Grégoire de Naziance attribue à quelques gouttes de sang la même efficacité qu'au corps dans son intégrité.

Mais en ce qui concerne le sang du Christ, il y avait une réticence. On vénérait davantage les objets qui ont été en contact avec son sang, ou tout simplement avec sa Passion. En tout cas, on peut constater que les reliques du sang du Christ sont souvent associées à d'autres reliques ou images miraculeuses: à Reichenau, le Saint Sang se trouve dans un reliquaire de la Croix; à Mantoue, on découvre dans une église le sang du Christ en même temps que le corps de Longin; le Volto Sancto arrive miraculeusement à Lucques avec deux ampoules du sang du Christ (dont une sera donnée à Luni). Les plus célèbres miracles du sang du Christ du haut Moyen Âge sont ceux du sang miraculeux qui coule des images attaquées comme l'icône du Christ à Béryte. De l'image du Christ, transpercé par un Juif, a jailli du sang que l'on a ensuite diffusé par l'envoi des ampoules. Cette image a d'ailleurs joué un rôle dans la querelle de l'iconoclasme en Orient, figurant parmi les preuves qui légitimaient la vénération des images. Mais aussi, en Occident, dans une chronique rédigée à la fin du $\mathrm{X}^{\mathrm{e}}$ siècle par un moine du Mont Soracte, on relate qu'en 846, lors du sac de Saint-Pierre, un Sarrasin a planté sa lance dans la poitrine de l'image du Seigneur qui se trouvait dans l'abside; aussitôt du sang en sortit.

Nous pouvons avec prudence avancer l'hypothèse selon laquelle c'est le concept de transsubstantiation qui a limité le culte du Saint Sang. Tout comme à Fécamp, ou dans les visions graaliennes dans les romans arthuriens, les mystères eucharistiques relativisaient la vénération qui s'adressait à la relique du sang. Si, par leur consécration, le pain et le vin se changent quotidiennement en chair et en sang du Christ, quel besoin de vénérer le sang-relique? D’autant plus qu'en guise de reliques, on pouvait placer des hosties consacrées dans l'autel!

Le véritable culte du Précieux Sang, puis celui du Sacré Cœur, se développent à la suite des changements de la sensibilité religieuse à partir des XII ${ }^{\mathrm{e}}$-XIII ${ }^{\mathrm{e}}$ siècles. Les raisons de cette évolution sont complexes: le développement de la religiosité individuelle, avec une intériorisation de la dévotion, qui mène à la méditation sur les souffrances du Christ, en faisant revivre sa Passion parfois jusqu'à l'apparition des stigmates sanglants sur le corps de quelques saints. Parallèlement à cela, 
l'exaltation de l'Eucharistie fait naître nombre de légendes où le sang du Christ coule réellement de l'hostie blessée, profanée.

Les tendances mystiques déplacent ensuite l'intérêt du Sang du Christ vers un nouvel objet de dévotion, le Sacré Cœur, qui correspond encore mieux à l'idée de l'union intime de l'être humain avec l'époux céleste.

Pendant que les images et les textes de la fin du Moyen Âge évoquent avec force la souffrance du Christ, les charmes pour arrêter le sang et guérir les plaies donnent une conception toute différente de la blessure du côté du Christ. Les formules dites de Longin mettent en relief le caractère miraculeux de la plaie du côté qui reste exempt de tout symptôme pathologique, ce trait miraculeux, d'allure docétiste, constituant le précédent légendaire pour la guérison du malade.

Sang réel du Christ, sang miraculeux des images, sang eucharistique: toutes ces sortes de sang sont les preuves de la présence divine vivante, leur dévotion rappelant, sous des formes variées, la symbolique du sacrifice du Christ pour le rachat de l'humanité. 